\title{
Establishment of a free-mating, long-standing (rossanat and highly productive laboratory colony of Anopheles darlingi from the Peruvian Amazon
}

\author{
Cuauhtémoc Villarreal-Treviño ${ }^{1 \dagger}$, Gissella M Vásquez ${ }^{2 * \dagger}$, Victor M López-Sifuentes ${ }^{2}$, Karin Escobedo-Vargas ${ }^{2}$, \\ Anibal Huayanay-Repetto ${ }^{2}$, Yvonne-Marie Linton ${ }^{3,4,5}$, Carmen Flores-Mendoza ${ }^{2}$, Andrés G Lescano ${ }^{6}$ \\ and Frederick M Stell ${ }^{2}$
}

\begin{abstract}
Background: Anopheles darlingi is the main malaria vector in the Amazon region and is among the most efficient malaria vectors worldwide. However, due to the lack of a well-established laboratory colony, key control-relevant aspects of the bionomics, behaviour, genetics, and vector-parasite relationships of An. darlingi remain unknown. Here, biological parameters that had been successful in initiating other Anopheles colonies were optimized and improved for An. darlingi, with the aim of establish a free-mating, stable, and highly productive laboratory colony.
\end{abstract}

Methods: Wild An. darlingi adult females were field collected from Zungarococha, Loreto Department, Peru $\left(03^{\circ} 49^{\prime}\right.$ $32.40^{\prime \prime} \mathrm{S}, 73^{\circ} 21^{\prime} 00.08^{\prime \prime} \mathrm{W}$ ), and taken to the NAMRU-6 Insectary in lquitos where $\mathrm{F}_{1}$ offspring were produced and reared. Natural copulation was successfully induced in $F_{1}$ adults under a thermoperiod of $30 \pm 1{ }^{\circ} \mathrm{C}$ during the day and $25 \pm 1{ }^{\circ} \mathrm{C}$ at night, and with a 30-min LED light stimulation period at dusk. Oviposition success was enhanced using egg-laying containers with a dark-coloured surface. Larval feeding regimes were standardized for optimal larval development. Optimized copulation induction methods were used to facilitate mating in An. darlingi until the $F_{10}$ generation. No copulation induction assistance was needed in subsequent generations.

Results: In 19 generations, the An. darlingi colony produced a total of 763,775 eggs; 441,124 larvae; 248,041 pupae; and 231,591 adults. A mean of 0.56 sexual encounters/female/cage ( $n=36$ cages) was recorded across the first ten generations $\left(F_{1}-F_{10}\right)$. A mean insemination rate of $54.7 \%\left(n=5,907\right.$ females) ranging from $43.6 \%\left(F_{2}\right)$ to $66.6 \%$ $\left(F_{10}\right)$ was recorded across nine generations $\left(F_{2}-F_{10}\right)$. Free-mating was casually observed in the $F_{8}$ generation, and subsequently confirmed in the $F_{9}$ and $F_{10}$ generations; comparable insemination rates and egg laying between stimulated (51.6\%, 12.9 eggs/female), and non-stimulated (52.3\%, 11.2 eggs/female) females were recorded. The time from egg to adult development ranged from 10 to 20 days. Moreover, the colony was relocated to a new laboratory within lquitos in the $\mathrm{F}_{14}$ generation without any noted changes in its productivity. By March 2015, the An. darlingi colony has been successfully reared to the $F_{26}$ generation.

Conclusions: This constitutes the first report of a free-mating, highly productive, and long-standing An. darlingi laboratory colony established through natural copulation induction, which will support critical malaria research. This rearing methodology may be a transferable, cost-effective alternative to labour-intensive forced mating practices widely used in maintaining other Anopheles colonies.

Keywords: Anopheles darlingi, Colony establishment, Natural copulation induction, Larval rearing, Amazon basin, Peru, Malaria vector

\footnotetext{
* Correspondence: gissella.vasquez@med.navy.mil

${ }^{\dagger}$ Equal contributors

${ }^{2}$ Department of Entomology, U.S. Naval Medical Research Unit No. 6 (NAMRU-6), Bellavista, Callao, Peru

Full list of author information is available at the end of the article
}

\section{Biomed Central}

(c) 2015 Villarreal-Treviño et al. This is an Open Access article distributed under the terms of the Creative Commons Attribution License (http://creativecommons.org/licenses/by/4.0), which permits unrestricted use, distribution, and reproduction in any medium, provided the original work is properly credited. The Creative Commons Public Domain Dedication waiver (http://creativecommons.org/publicdomain/zero/1.0/) applies to the data made available in this article, unless otherwise stated. 


\section{Background}

Anopheles darlingi is considered the most effective malaria vector in the Neotropical region $[1,2]$ and is responsible for most malaria transmission where it is found, including areas of high deforestation [3, 4]. This species efficiently transmits Plasmodium falciparum, Plasmodium vivax VK210, and P. vivax VK247 across Latin America [5-9], and its introduction to the northern Amazon has been linked to the considerable increase of P. falciparum cases in Peru in the 1990s $[10,11]$. In Peru, An. darlingi is the dominant malaria vector in the Loreto Department, which is the most affected malaria endemic region in the country. Anopheles darlingi is present in both rural villages in the vicinity of Iquitos (15-20 km west-southwest) where it comprises 67-99 \% of anophelines collected [12], and riverside camps along the Mazan river $(40-50 \mathrm{~km}$ northeast of Iquitos) where $99 \%$ of anophelines collected were identified as An. darlingi [13]. Interestingly, An. darlingi biting rates, and entomological inoculation rates (EIRs) differed considerably among these locations and are substantially higher at riverine sites [13], possibly linked to the observed malaria transmission heterogeneity in the Peruvian Amazon. Therefore, knowledge of the biology, behaviour, and vectorial capacity of this vector is crucial for understanding malaria transmission dynamics in the Amazon region, as well as for developing and implementing effective malaria vector-targeted control strategies. Laboratory colonies of An. darlingi reared up to the F9 generation have been reported before, producing less than 3,000 mosquitoes per generation $[14,15]$ and without description of long-term continuity. The lack of a highly-productive and well-established An. darlingi laboratory colony that can continuously provide large numbers of mosquitoes has limited these critical studies.

Previous reports regarding the establishment of a laboratory colony of An. darlingi [16-19] did not explain the main factors triggering sexual behavior and regulating colony productivity in this species. This historically limited replication of the methodology used and the establishment of new laboratory colonies. For example, low sexual activity resulting in limited oviposition in $\mathrm{F}_{2}$ adult females made establishment of a colony in São Paulo unsuccessful [19]. Recently, a method developed for Anopheles pseudopunctipennis was used for colonization of $A$. darlingi in the laboratory [20], yet very low densities of laboratory-reared adults across six generations (1,618 adults per generation on average) were reported. Moreover, critical characterization of sexual behaviour or parameters demonstrating adaptation of $\mathrm{F}_{1}$ to laboratory conditions and colony stability has not been reported to date [16-20]. This is an area with limited to no evidence. There is only one report from an An. darlingi markrecapture study describing that sexual activity in field populations occurs in the evening shortly after the sunset and near human dwellings, yet no male swarms were observed despite the finding of inseminated recaptured females [20].

Thus, the main factors stimulating An. darlingi sexual behaviour and oviposition in the field and under laboratory conditions still remain unknown [14, 21]. Limited success of copulation induction in artificial environments such as an insectary, where adults are generally confined to rearing cages $[19,22,23]$, remains the major blockage to colony establishment in An. darlingi. Forced copulation technique was explored in An. darlingi colony establishment, $[24,25]$. Not only is this very difficult and time-consuming [14] but was also unsuccessful for $A n$. darlingi with a very low percentage of $\mathrm{F}_{1}$ females (1\%) laying eggs (Escobedo and Huayanay, personal observation). The inability to induce copulation, in addition to the lack of an established method for mass larvae rearing, considerably impeded the establishment of a long-standing, highly productive An. darlingi laboratory colony.

Therefore, a natural copulation induction technique that has effectively worked for colonization of An. pseudopunctipennis and other anophelines including $A n$. darlingi in Mexico although only until the $\mathrm{F}_{9}$ generation $[14,22]$, was evaluated, adapted and optimized. Also, conditions favoring egg-laying in gravid females were standardized and larvae rearing was improved by providing a diet that meets nutritional needs, has appropriate particle size, and is easily digestible $[14,22]$. This combined approach has allowed to develop an effective method to establish and mass-rear An. darlingi under insectary conditions. The methodology used is described in all its complexity and detail to contribute to the development of other laboratory colonies across the Americas and for potential replication in other Anopheles species.

\section{Methods}

\section{Study design}

The $\mathrm{F}_{1}$ offspring of wild-caught An. darlingi females were used to evaluate and optimize techniques for natural copulation stimulation, oviposition induction, and larvae rearing. Sexual behaviour was examined by direct observation of mating across 10 generations $\left(\mathrm{F}_{1}-\mathrm{F}_{10}\right)$, and video recording of adult behaviour during mating in $F_{1}$ and $F_{14}$ individuals. Successful mating was assessed by estimating the rate of inseminated females via spermatheca dissections across 10 generations $\left(\mathrm{F}_{1}-\mathrm{F}_{10}\right)$. Free-mating was confirmed by comparing the rate of insemination and egg-laying between stimulated and nonstimulated $\mathrm{F}_{9}$ and $\mathrm{F}_{10}$ adults. Copulation induction was deemed unnecessary from the $F_{11}$ generation onwards. 


\section{Anopheles darlingi field collection and production of $F_{1}$ adults}

Adult females were collected in February 2013 (rainy season) in the community of Zungarococha (03 $49^{\prime}$ $\left.32.40^{\prime \prime} \mathrm{S}, 73^{\circ} 21^{\prime} 00.08^{\prime \prime} \mathrm{W}\right), 18 \mathrm{~km}$ southwest of Iquitos, Peru. Active transmission of $P$. falciparum and $P$. vivax historically occurs in this area [26], where An. darlingi has been incriminated as the main vector [12]. Adult females were captured hourly from 18:00 to 22:00 using protected human landing collections [25]. Anopheles darlingi females collected were placed in carton cups $(250 \mathrm{ml})$ covered with nylon mesh, provided $10 \%$ sugar solution and taken to the NAMRU-6 insectary in Iquitos. Here, cow, or chicken blood was offered through glass membrane feeders ( $3.8 \mathrm{~cm}$ outer diameter) to induce oviposition and obtain $F_{1}$ adults following previously described procedures [21, 27]. Briefly, one wing of each blood-engorged female was cut and then females were placed individually in vials for egg laying. Eggs were inundated with water within $24 \mathrm{~h}$ and placed in plastic trays $(26.5 \times 16.5 \mathrm{~cm})$. Larvae were transferred into plastic trays containing a mix of water from natural breeding sites and filtered water and fed a mixture of wheat flour and fish-meal with quantities increasing with larval development from $0.14 \mathrm{mg} /$ larva (first instar) to $0.5 \mathrm{mg} /$ larva (fourth instar). Pupae were transferred into plastic containers with water (200 pupae/container) and placed in screen cages for adult eclosion. $F_{1}$ adults were used in natural copulation induction assays and for optimization of oviposition and larvae rearing. Morphological species identification of wild-caught and $\mathrm{F}_{1}$ adult Anopheles darlingi was conducted using keys for Neotropical Anopheles $[28,29]$. Molecular verification of $\mathrm{F}_{10}$ An. darlingi adults was confirmed using mitochondrial cytochrome $c$ oxidase I (COI) gene sequences, following the standard protocol of the Mosquito Barcoding Initiative [30].

\section{Standardization of the natural copulation induction technique}

The natural copulation induction technique developed for An. pseudopunctipennis [22] was initially evaluated against laboratory reared $A n$. darlingi $\mathrm{F}_{1}$ adults obtained in February 2013. As the appropriate/critical space for copulation, sugar source finding, and mosquito density were unknown, two sizes of screened cages, medium $(46 \times 46 \times 46 \mathrm{~cm})$ and large $(61 \times 61 \times 61 \mathrm{~cm})$ were evaluated simultaneously following modifications on documented environmental conditions known to trigger natural copulation in An. pseudopunctipennis [22]. Briefly, 2-5 day old $A n$. darlingi $\mathrm{F}_{1}$ males and females were placed in the two sizes of screened cages: 1,500 adults in the medium cage (1:1 females to males) and 2,400 adults in the large cage (1:1 females to males), and fed ad libitum with honey-water solution (10\%). Screened cages were kept in a room with controlled temperature that was maintained at $30 \pm 1{ }^{\circ} \mathrm{C}$ during the day (07:00 to $\left.19: 00\right)$ and at $25 \pm 1^{\circ} \mathrm{C}$ during the night (19:00 to 07:00) and under a 12:12 light to dark photoperiod. Relative humidity was not controlled and ranged from 63 to $80 \%$ following natural conditions in the city of Iquitos. $F_{1}$ adult mosquitoes were exposed to a beam of a Light-Emitting Diode (LED) projected with a flashlight of $1.5 \mathrm{~W}$ placed $50 \mathrm{~cm}$ away from the cage. Three artificial 15-min periods of light exposure interjected by 5 -min dark intervals were conducted at dusk for five consecutive days in a bid to increase sexual arousal and trigger natural copulation. Light exposure was carried out in complete silence to avoid disruption of mosquito sexual behaviour. Three days after the first copulation induction, $\mathrm{F}_{1}$ female mosquitoes were offered commercially purchased animal blood (either cow or chicken blood) via membrane feeders for 30-40 min. Blood meals were offered every two days for a period of 15 days from 1900 to $2000 \mathrm{~h}$ (recently induced females) and from 0900 to1030 h (ovipositing females); chicken blood was given twice during the first gonotrophic cycle (2-3 days) and either chicken or cow blood was provided at each subsequent feeding event. Fecundity (number of eggs laid per female) between females from cages that received only one type of blood versus those that received two types of blood was compared. In addition, wing length ( $\mathrm{mm}$ ) of a sample of females (10) per generation $\left(\mathrm{F}_{1}-\mathrm{F}_{10}\right)$ was measured with a stereoscope (MEIJI EMZ-13, Saitama, Japan) at 30× magnification to examine changes in body size across generations, which could be linked to fecundity [31, 32]. These standardized copulation induction conditions were used to rear $A n$. darlingi up to the $\mathrm{F}_{10}$ generation but using large cages only based on results from the cage-size evaluation.

\section{Assessment of An. darlingi sexual behavior and natural copulation under laboratory conditions}

Direct observation of natural copulation in $\mathrm{F}_{1}$ mosquitoes was conducted in both medium and large cages during all three light stimulation periods for seven consecutive days. Mating pairs (copulations) were counted as mating pairs who encountered at flight and fell on the cage floor. Results are presented as total numbers of copulations/day/ cage size as well as number of copulations/100 females, to account for the differences in total numbers of females placed in the medium and large cages. In subsequent generations (up to $F_{10}$ ), the total number of copulations/ female/cage was recorded but for only five days. Sexual behaviour of $F_{1}$ and $F_{14}$ males and females was recorded with a Nikon ${ }^{\circ}$ D5100 video camera. The video was reviewed frame by frame in iPhoto'11 version 9.3 on a $1600 \mathrm{MHz}$ iBook computer with an 11-in. video screen (Apple Computers, Cupertino, CA). Copulation time was recorded and sexual behaviour traits noted. 
Insemination rates were estimated by dissecting the spermathecae of a representative sample of adult females collected from each cage across 9 generations $\left(F_{2}-F_{10}\right)$, following the WHO standard methods [25]. Fallen females were collected daily for a period of 5-17 days, starting one day after the first day of copulation induction.

Apparent copulation in the absence of stimulation was first observed in the $\mathrm{F}_{8}$ generation. Direct observation of copulation in adults not stimulated by light and thermoperiod was conducted between 18:30 and 19:30 for the $F_{9}$ and $F_{10}$ generations. Free-mating behaviour was evaluated by comparing insemination rates per cage and egg-laying rates per female in those copulation-induced females versus non-stimulated controls. Insemination rates were determined as described above; egg-laying rates were estimated by dividing the total number of eggs laid per cage by the total number of females per cage.

\section{Optimization of An. darlingi oviposition and larvae rearing}

Preliminary observations of oviposition preference of blood-fed $\mathrm{F}_{1}$ adult females were conducted in the NAMRU-6 Iquitos insectary, comparing light-coloured versus dark-coloured oviposition surfaces. Four white plastic trays $(32 \times 18 \times 5 \mathrm{~cm})$ and four additional identical trays covered with black plastic were filled with filtered water and placed into cages containing 2,000 $F_{1}$ adults. Female attraction to each colored tray and oviposition rates were recorded. It was subsequently evaluated whether the addition of native aquatic plants (Ceratopteris pteridoides) commonly found in breeding sites would increase oviposition. Environmental conditions were identical for oviposition and natural copulation induction as eggs were collected in the same environment where cages with adults were kept. No honey solution was provided during the oviposition period (15 days). $\mathrm{F}_{1}$ eggs (c. 500) were transferred into larger plastic trays $(37 \times 25.5 \times 5.5 \mathrm{~cm})$ containing $1-1.5 \mathrm{~L}$ of filtered, dechlorinated water by directly pouring water from eggcollecting trays. These trays were kept at $28 \pm 1{ }^{\circ} \mathrm{C}, 66 \pm 1$ $\mathrm{RH} \%$ and at a 12:12 light to dark photoperiod, conditions favourable for larval eclosion. In subsequent generations, eggs were collected on strips of filter paper $(2 \mathrm{~cm}$ wide) lining tray edges to prevent egg damage.

Approximately $500 \mathrm{~F}_{1}$ first-instar larvae were reared in plastic trays $(37 \times 25.5 \times 5.5 \mathrm{~cm})$ until development of third-instar larvae. Third-instar larvae were divided into two trays so that a mean density of 250 larvae per tray was maintained until development of pupae. Larvae were fed commercially available rodent food (Laboratory Rodent Diet 5001, LabDiet ${ }^{\circ}$, St. Louis, MO) containing $23.0 \%$ protein, $4.5 \%$ fat, and $6.0 \%$ crude fiber. Optimal feeding amount and frequency of dry rodent food fed were assessed and optimized for each larval instar as previously described [14, 22].

When larval mortality was higher than average, microscopic examination $(40 \times)$ of water samples was performed from trays to determine the presence of bacteria, fungi and protists. Selective, and differential media (MacConkey agar, xylose lysine deoxycholate agar, and thiosulfate citrate bile salts sucrose agar) were used to differentiate bacteria.

Standardized oviposition and larvae rearing procedures have been used to rear $A n$. darlingi up to the $\mathrm{F}_{26}$ generation. The colony had to be relocated to another NAMRU-6 facility in Iquitos while rearing the $\mathrm{F}_{14}$ generation, adaptation to laboratory rearing facilitated this process.

\section{Research ethics}

Human landing collections were conducted following NAMRU-6's security protocol, including explanation of risks, safety, healthcare, prophylaxis, and in case of disease, the appropriate care. Mosquito blood feeding using membrane feeders was conducted following the standard operating procedures of NAMRU-6.

\section{Statistical analyses}

Reductions in larval mortality across generations were evaluated with Spearman's Rho non-parametric correlation tests. Changes in egg, larvae and pupae productivity, and reductions in mortality associated with natural copulation were tested using Wilcoxon rank-sum (MannWhitney) non-parametric tests. Independent two sample $t$-tests assuming unequal variances (separate-variances $t$-tests) were performed for mean comparison of insemination rates and number of eggs laid by stimulated versus not-stimulated females; number of copulations/100 females in large versus medium cages; and number of eggs laid on black trays versus white trays. A Least Significant Difference (LSD) test was performed for mean comparison of female wing length. All analyses were conducted with Stata v13.0 (StataCorp, College Station, TX, 2013) except the LSD test that was conducted with SAS 9.4 (SAS Institute Inc., Cary, NC, 2002-2013). P-values $<0.05$ were considered statistically significant.

\section{Results}

Anopheles darlingi colony establishment through natural copulation induction

The offspring ( $\mathrm{F}_{1}$ individuals) of 878 wild-caught adult females from Zungarococha, Loreto served to establish an $A n$. darlingi colony. Species identity of the laboratory colony was confirmed both by morphological analysis of wild-caught, $F_{1}$ and $F_{10}$ adults, and by DNA barcoding an $\mathrm{F}_{10}$ adult. The COI sequence data $(658 \mathrm{bp}$, corresponding to the barcode region) was compared with 
expertly identified specimens in the databank of the Mosquito Barcoding Initiative (Barcode of Life Database, BOLD), and those available in GenBank. The colony sequence (GenBank accession number KP193458) showed 99.6-100\% identity with other $A n$. darlingi sequences from Peru, Bolivia, and Brazil in the Barcode of Life Database (BOLD) [33]. Comparison with published GenBank entries revealed highest identity with JF923693 from Acrelandia, Brazil, (99.34\%) and 99.2\% and $98.8 \%$ identity with GQ918272 and GQ918273, the complete mitochondrial genome from the northern and southern lineages of $A n$. darlingi, respectively [34].

During first attempts of applying and optimizing thermoperiod and light exposure regimes used for $A n$. pseudopunctipennis [22] in February 2013, it was observed, and digitally recorded for the first time $A n$. darlingi natural copulation under laboratory conditions. This significant milestone is publicly available in Additional file 1A, and documents the sexual behavior of $A n$. darlingi for the first time. In this first experiment, copulation induction was tested in medium and large screened cages. In both cage sizes, copulation increased from day 1 to day 4 , and then decreased as shown by the daily number of natural copulations (Table 1) and the number of copulations per $100 \mathrm{fe}-$ males (Fig. 1). In the medium-sized cage, the highest number of natural copulations was recorded on day 4 (15 total: two per 100 females), and the lowest number on day 6 (four total: 0.5 per 100 females). Copulation was substantially higher in the large cage on days 2-4 (60-100 total: $5-8$ per 100 females). The number of copulations per 100 females (mean \pm standard deviation) on days 2-4 was significantly higher in the large cage $(6.3 \pm 1.8)$ than in the medium cage $(1.5 \pm 0.5)$ ( $t$-test: $p=0.0238)$. Therefore, large cages with a density of approximately 2,500 adult

Table 1 Number of natural copulations recorded for different thermoperiod/light induction periods for An. darlingi enclosed in cages of two different sizes (medium $=46 \times 46 \times 46 \mathrm{~cm}$; large $=61 \times 61 \times 61 \mathrm{~cm}$ ) and with different adult densities

\begin{tabular}{|c|c|c|}
\hline \multirow{3}{*}{$\begin{array}{l}\text { Period of natural copulation } \\
\text { induction (days) }\end{array}$} & \multirow{3}{*}{$\begin{array}{l}\text { Medium cage } \\
\text { 1,500 adults } \\
(1: 1 \delta: \text { o })\end{array}$} & \multirow{3}{*}{$\begin{array}{l}\text { Large cage } \\
2,400 \text { adults } \\
(1: 1 \quad \delta: 9)\end{array}$} \\
\hline & & \\
\hline & & \\
\hline 1 & 5 & 10 \\
\hline 2 & 8 & 65 \\
\hline 3 & 10 & 60 \\
\hline 4 & 15 & 100 \\
\hline 5 & 8 & 4 \\
\hline 6 & 4 & 11 \\
\hline 7 & 5 & 5 \\
\hline Total number of copulations & 55 & 255 \\
\hline
\end{tabular}

$F_{1}$ adults tested were 2-3 days old. Observations were conducted in the NAMRU-6 insectary in lquitos, Peru mosquitoes were used since to provide the best conditions for natural copulation in subsequent generations. Copulation induction was conducted only for five days up to the $\mathrm{F}_{10}$ generation when free-mating was confirmed.

The percent insemination rate recorded in An. darlingi $\mathrm{F}_{2}$ adult females was $43.6 \pm 5.4 \%$. This insemination rate was higher when compared to those previously recorded in $\mathrm{F}_{1}$ adult females reared at the NAMRU-6 Insectary [21] that had not been stimulated, $2 \pm 2.8 \%$, or stimulated with light only, $5 \pm 1.4 \%$ (Fig. 2). Copulation induction using a combination of light and thermoperiod as stimuli was more effective in triggering adult copulation as evidenced by the higher insemination rate recorded relative to those recorded with no stimulus or stimulated by light only.

\section{Sexual behavior, copulation, and insemination rates in colonized Anopheles darlingi}

General aspects of the $A n$. darlingi sexual behaviour were examined frame by frame in a $17-$ min video recorded during copulation induction of $\mathrm{F}_{1}$ adults (Additional file 1A). Forty-five natural copulations at the rate of approximately 2.6 copulations per minute were recorded. Males and females mated in midair and copulation lasted on average $1.9( \pm 2.2)$ seconds, ranging from 1 to $10 \mathrm{~s}$. In nearly all observed copulations (97.7 \%), the mating pair fell on the cage floor and $84.1 \%$ (37 of 44), where they remained for less than a second, before separating, and flying away. The remaining seven couplets stayed together for over a second. After copulation ended, males, and females separated from each other, and females immediately flew away. Only a fraction of males $(51.1 \%)$ that had separated from females stayed on the cage floor for $6.1( \pm 7.8)$ seconds on average in apparent rest or recovery, and then flew away. Flight height above cage floor, wing flapping frequency, copulation in midair, length of copulation, in $\mathrm{F}_{14}$ adults was similar to that of $\mathrm{F}_{1}$ adults, but copulation rates were 7.7 times higher, at c. 20 copulations per minute (Additional file 1B).

The mean number of copulations/female/cage $(n=36)$ increased significantly across the first ten generations (Spearman's rho $=0.66 ; p<0.0001$ ) (Fig. 3). The lowest mean number of copulations/female/cage was recorded for the $F_{1}$ generation $(0.1 \pm 0.1)$ and the highest for the $F_{8}$ generation $(1.1 \pm 0.5)$. The mean insemination rate $(\%)$ recorded per cage $(n=34 ; 45-367$ females sampled per cage) increased at a marginal significant rate (Spearman's rho $=0.26 ; p<0.1340)$ across the first nine generations $\left(\mathrm{F}_{2}-\mathrm{F}_{9}\right)$ (Fig. 3). The lowest mean insemination rate was recorded for the $F_{2}$ generation $(43.6 \pm 5.4)$, while the highest for the $F_{8}$ generation $(67.4 \pm 6.1)$. Mean insemination rates have reached $>50 \%$ since the $\mathrm{F}_{6}$ generation.

In females from the first ten generations, egg laying per female did not differ between females offered one 


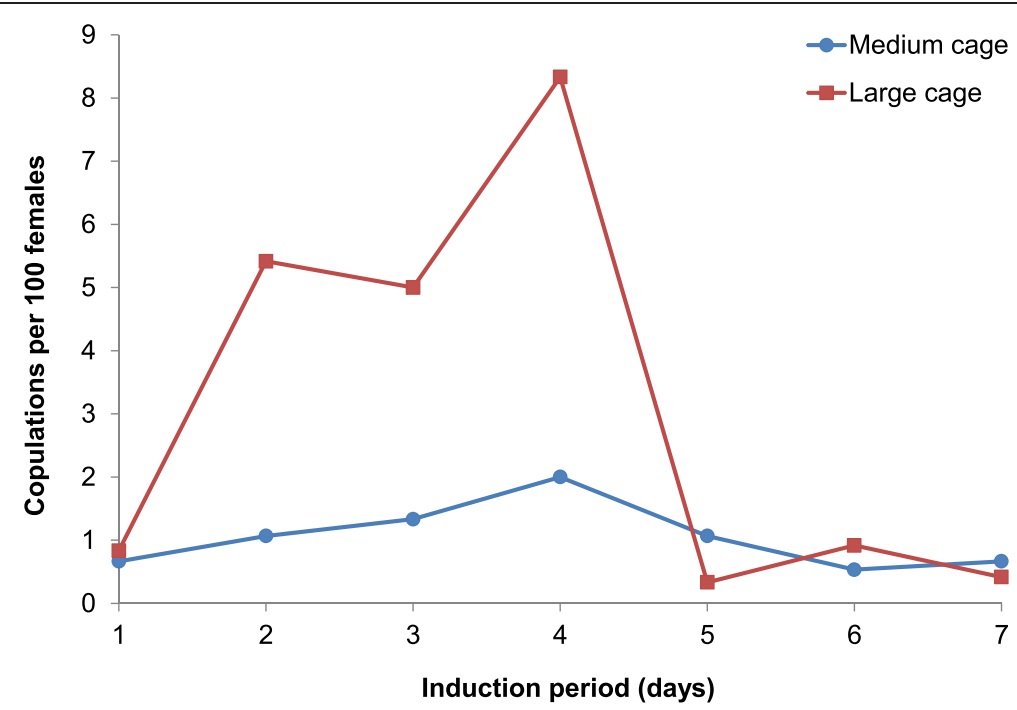

Fig. 1 Copulation induction period and number of natural copulations observed per 100 An. darlingi $\mathrm{F}_{1}$ adult females kept in medium (46 $\times 46 \times 46 \mathrm{~cm}$ ) and large $(6161 \times 61 \mathrm{~cm})$ cages under laboratory conditions in the NAMRU-6 insectary in lquitos, Peru

type of animal blood (17.2 \pm 14.9 eggs/female) and those given two choices of animal blood $(26.5 \pm 22.7 \mathrm{eggs} / \mathrm{fe}-$ male) ( $t$-test: $p=0.0808)$. However, significant differences were detected in wing length of females from the $\mathrm{F}_{1}-\mathrm{F}_{10}$ generations and the Zungarococha field population $(F=6.40 ; p<0.0001)$, with the smallest wing length $(2.70 \pm 0.11 \mathrm{~mm})$ recorded in the $F_{2}$ generation and the largest $(3.04 \pm 0.13 \mathrm{~mm})$ for wild-caught females. $F_{9}, F_{10}$, and wild-caught females had larger wings than females from generations $F_{1}-F_{8}$ (LSD $p<0.05$ ), suggesting that female fecundity in the first eight generations could have been lower than that of field populations, yet comparable in later generations [31, 32].

The occurrence of free-mating was first recorded in the $\mathrm{F}_{8}$ generation upon occasional observation of copulation in the absence of stimulation, therefore direct observation of free mating and comparison of insemination rate and egg laying between stimulated and non-stimulated females were conducted in the $F_{9}$ and $F_{10}$ generations. Moreover, there were no significant differences in the mean insemination rates per cage recorded for stimulated $(51.6 \pm 12.7 \%)$ and non-stimulated $(52.3 \pm 2.9 \%)$ females from the $F_{9}$

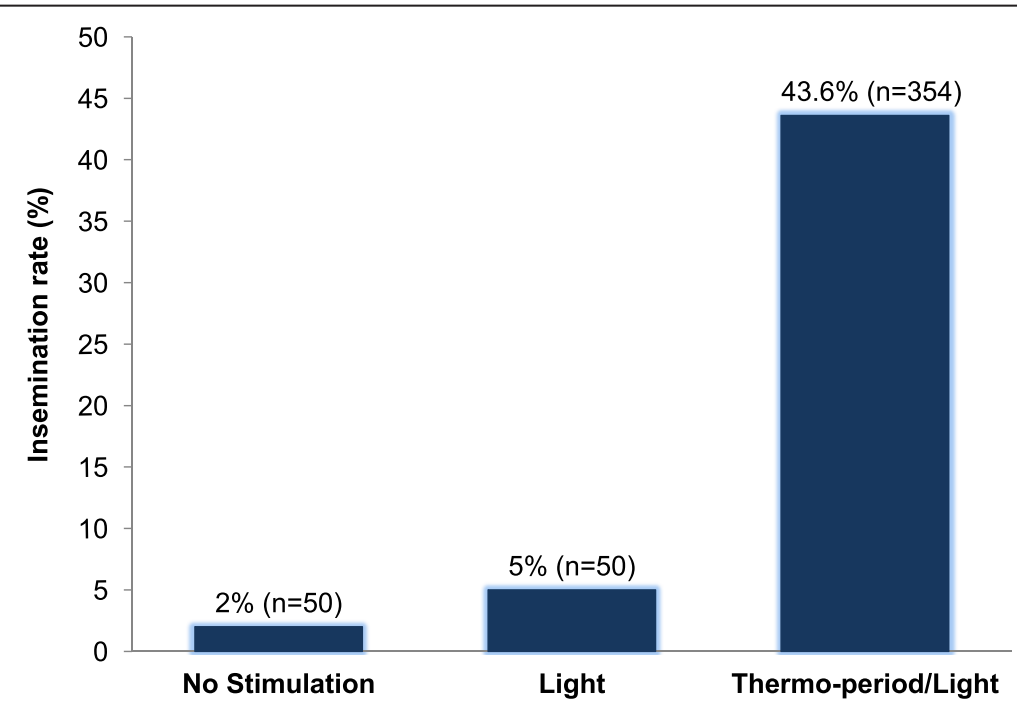

Fig. 2 Anopheles darlingi insemination rates (\%) recorded for adult females that received i) no copulation stimulation, ii) copulation stimulation using light only, and iii) copulation stimulation using thermoperiod and light. Copulation data without stimulation, and light only copulation stimulation data were taken from [22]. Insemination rates estimated under no stimulation and light only conditions were recorded in $\mathrm{F}_{1}$ adult females, and under thermoperiod/light in $F_{2}$ adult females. Adult females were reared in the NAMRU-6 insectary in lquitos, Peru 


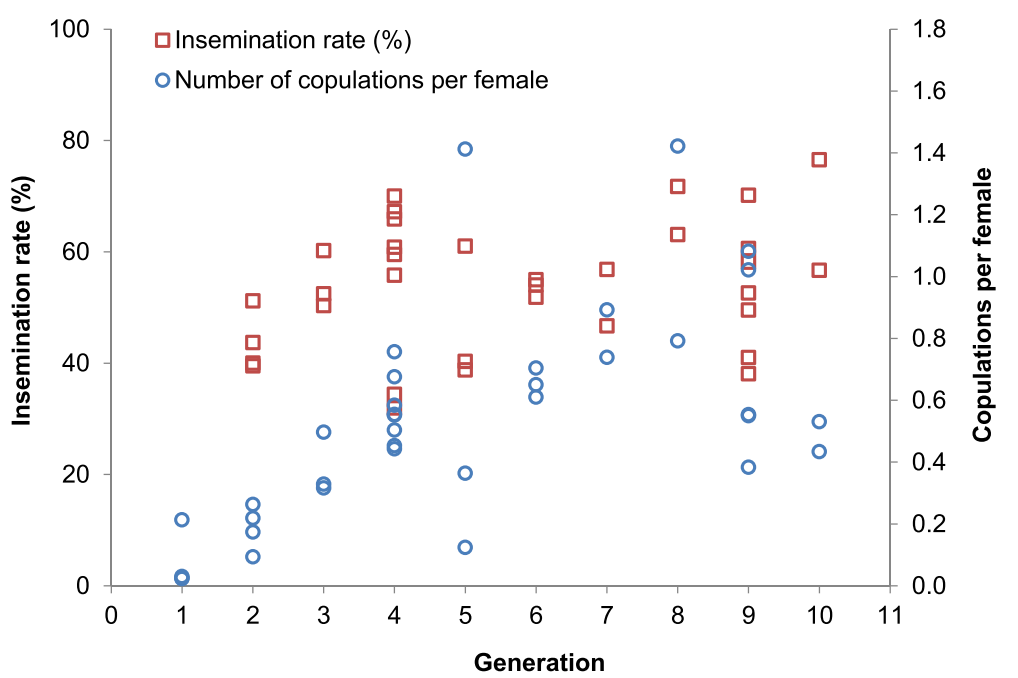

Fig. 3 Anopheles darlingi copulations per female and insemination rates $(\%)$ recorded across 10 generations $\left(F_{1}-F_{10}\right)$ reared in the NAMRU-6 insectary in lquitos, Peru

and $\mathrm{F}_{10}$ generations $(t$-test: $p=0.4563)$. Likewise, the numbers of eggs laid per stimulated $(12.9 \pm 4.4)$ and non-stimulated (11.2 \pm 7.3$)$ females did not significantly differ ( $t$-test: $p=0.2896)$. These results confirmed that this An. darlingi colony was self-mating, therefore, copulation induction procedures were ceased from the $\mathrm{F}_{11}$ generation. The colony has continued through the $\mathrm{F}_{26}$ generation with an increase in egg and adult production.

\section{Productivity and continuity of the Anopheles darlingi laboratory colony}

Oviposition preference experiments showed that the mean number of eggs laid on trays covered with black plastic $(84.5 \pm 24.4)$ was significantly higher than those laid on white trays $(16 \pm 2.2)(t$-test: $p=0.0056)$, suggesting that $A n$. darlingi females preferred to lay their eggs on darker strata. It was determined that $20 \mathrm{mg}$ per tray given at a frequency of $1,2,3$, and 4 times per day for first, second, third, and fourth instars respectively, was optimal for larval development and also reduced water contamination due to bacterial growth on excess food. The estimated number of larvae per tray was 500 for first and second instars, and 250 for third, and fourth instars. These oviposition conditions and larval feeding regimes have been used for continuous rearing of colonized An. darlingi.

The An. darlingi colony has been maintained for 26 generations (February 2013 - March 2015), indicating successful adaptation to insectary conditions. An additional file shows the An. darlingi immature and adult rearing rooms in the NAMRU-6 Insectary in Iquitos (see Additional file 2). Data recorded across 19 successive generations $\left(\mathrm{F}_{1}-\mathrm{F}_{19}\right)$ strongly demonstrate the overall steadiness and increasing productivity of this free-mating colony as shown by the production of a total of 763,775 eggs; 441,124 larvae; 248,041 pupae; and 231,591 adults (data collected from February 2013 to June 2014) (Table 2). It is possible that the colony's free-mating nature and relative stability facilitated its re-establishment in this new facility where its rearing was continued up to the $\mathrm{F}_{26}$ generation to date.

During this laboratory adaptation process, total numbers of eggs produced increased across generations (Fig. 4), although the percent of unhatched eggs varied (Fig. 5). The total number of eggs laid per generation ranged from 4,950 to 116,465 , and the percent of unhatched eggs ranged from $4.0 \%$ to $61.3 \%$ (Table 2). The rate of unhatched eggs was above $60 \%$ in a few generations $\left(F_{3}, F_{6}, F_{11}\right.$, and $\left.F_{16}\right)$, but below $50 \%$ for all other generations (Fig. 5). $F_{13}$ eggs were checked under the micro stereoscope five days after placement in hatching trays to observe whether they had opened opercula and verify egg fertilization. Observations made on $\mathrm{F}_{13}$ eggs with a closed operculum (unhatched eggs) indicated that these were mainly unfertilized eggs rather than unviable/ damaged fertilized eggs, which may be in part linked to insemination rates recorded $(54.7 \pm 7.9 \%$, mean across nine generations).

The total number of larvae produced per generation ranged from 3,500 to 75,000 with a mean larva-pupa mortality of $39.7 \pm 29.6 \%$ (Table 2). Larval mortality was less than $10 \%$ in the first three generations, but increased in subsequent generations with peaks $(>80 \%)$ recorded for $F_{5}, F_{14}$, and $F_{17}$ larvae (Fig. 5). Larval mortality was most likely associated with bacterial infections (Enterobacter sp., Serratia marcescens, Pseudomonas sp.) as observed in $\mathrm{F}_{4}$ larvae, and / or water contamination by fungi and protozoa as observed when rearing $F_{5}, F_{14}$, and $\mathrm{F}_{17}$ larvae. In addition, rodent food availability was 
Table 2 Total numbers of eggs, larvae, pupae, and adults; and percentage of unhatched eggs, larval, and pupal mortality of An. darlingi reared for 19 generations $\left(F_{1}-F_{19}\right)$ in the NAMRU-6 insectary in lquitos, Peru

\begin{tabular}{|c|c|c|c|c|c|c|c|c|c|}
\hline Generation & $\begin{array}{l}\text { No. of } \\
\text { cages }\end{array}$ & $\begin{array}{l}\text { Copulation induction } \\
\text { period (days) }\end{array}$ & Total eggs & $\begin{array}{l}\text { Unhatched } \\
\text { eggs (\%) }\end{array}$ & Total larvae & $\begin{array}{l}\text { Larva-pupa } \\
\text { mortality (\%) }\end{array}$ & Total pupae & $\begin{array}{l}\text { Pupa-adult } \\
\text { mortality (\%) }\end{array}$ & Total adults ${ }^{a}$ \\
\hline 1 & 4 & 5 & 5,000 & 4.0 & 4,800 & 4.2 & 4,600 & 8.0 & 4,230 \\
\hline 2 & 4 & 5 & 10,000 & 19.4 & 8,063 & 0.9 & 7,993 & 5.0 & 7,593 \\
\hline 3 & 3 & 5 & 10,590 & 61.3 & 4,095 & 5.8 & 3,859 & 4.0 & 3,704 \\
\hline 4 & 8 & 5 & 29,380 & 48.9 & 15,000 & $44.5^{b}$ & 8,327 & 1.3 & 8,216 \\
\hline 5 & 3 & 5 & 35,419 & 22.0 & 27,616 & $93.3^{c}$ & 1,845 & $34.0^{c}$ & 1,218 \\
\hline 6 & 3 & 5 & 14,560 & 55.0 & 6,550 & 51.4 & 3,184 & 6.2 & 2,987 \\
\hline 7 & 2 & 5 & 18,790 & 33.5 & 12,500 & 47.6 & 6,544 & 8.3 & 6,000 \\
\hline 8 & 2 & 5 & 4,950 & 29.3 & 3,500 & 54.7 & 1,586 & 6.4 & 1,485 \\
\hline 9 & 7 & $0 / 5^{d}$ & 24,000 & 47.9 & 12,500 & 35.4 & 8,073 & 7.6 & 7,460 \\
\hline 10 & 10 & $0 / 3^{e}$ & 43,810 & 44.1 & 24,500 & 11.8 & 21,619 & 6.5 & 20,205 \\
\hline 11 & 7 & 0 & 46,600 & 57.1 & 20,000 & 13.3 & 17,339 & 4.9 & 16,485 \\
\hline 12 & 6 & 0 & 45,450 & 45.0 & 25,000 & 4.8 & 23,790 & 8.9 & 21,664 \\
\hline 13 & 5 & 0 & 49,130 & 49.1 & 25,000 & 40.1 & 14,976 & 9.0 & 13,629 \\
\hline 14 & 8 & 0 & 61,750 & 51.4 & 30,000 & $86.1^{c}$ & 4,183 & $21.4^{c}$ & 3,289 \\
\hline 15 & 6 & 0 & 54,000 & 55.6 & 24,000 & 46.0 & 12,951 & 8.9 & 11,801 \\
\hline 16 & 9 & 0 & 116,465 & 59.6 & 47,000 & 54.7 & 21,272 & 7.0 & 19,773 \\
\hline 17 & 6 & 0 & 57,100 & 29.9 & 40,000 & $82.2^{c}$ & 7,130 & 11.0 & 6,345 \\
\hline 18 & 6 & 0 & 53,880 & 33.2 & 36,000 & 67.2 & 11,820 & 6.5 & 11,057 \\
\hline 19 & 7 & 0 & 82,901 & 9.5 & 75,000 & 10.7 & 66,950 & 3.7 & 64,450 \\
\hline TOTAL & & & 763,775 & & 441,124 & & 248,041 & & 231,591 \\
\hline MEAN (SD) & & & $40,199(28,594)$ & $39.8(17.1)$ & $23,217(17,756)$ & 39.7 (29.6) & $13,055(14,765)$ & $8.9(7.3)$ & $12,189(14,202)$ \\
\hline $95 \% \mathrm{Cl}$ & & & $26,417-53,981$ & $31.5-48.0$ & $14,659-31,775$ & $25.5-54.0$ & $5,938-20,171$ & $5.4-12.4$ & $5,344-19,034$ \\
\hline
\end{tabular}

${ }^{a}$ Mean sex ratio (male:female) across generations is 1:1

bresence of Enterobacter sp. and Serratia marcescens

'Presence of fungus and protists

${ }^{d}$ Copulation induction performed for 5 days only in 4 cages

${ }^{\mathrm{e}}$ Copulation induction performed for 3 days only in 2 cages

limited for $\mathrm{F}_{5}$ larvae, and was temporarily replaced by spirulina (200 mg, first and second instars), and Koi food (120-300 mg, third, and fourth instars). Rodent food was available again for the $F_{6}$ generation, which helped reduce larval mortality in this generation. A significant reduction in larval mortality $(p=0.0020)$ was recorded after the contamination/diet issues in the $F_{5}$ generation but only up to the $F_{12}$ generation where larval mortality rates were comparable to the lowest rates recorded in the first three generations (Fig. 5). However, water contamination again led to high mortality in $\mathrm{F}_{14}$ and $\mathrm{F}_{17}$ immatures. To reduce water contamination issues, larvae rearing trays were covered with plastic screens to avoid exposure to pathogens commonly found in the environment.

Pupae production ranged from 1,586 to 66,950 with a mean pupal mortality of $8.9 \%( \pm 7.3 \%)$ that has been under $12 \%$ in all generations except for the $F_{5}(34.0 \%)$ and $\mathrm{F}_{14}(21.4 \%)$, due to presence of fungus and protists (Table 2, Fig. 5). The total number of adults produced per generation also varied with the lowest $(1,218)$ in the $F_{5}$ and the highest $(64,450)$ in the $F_{19}$ (Table 2, Fig. 4).

Overall, egg, and adult productivity increased considerably after the $\mathrm{F}_{8}$ generation $(p=0.002$ and $p=0.0179$, respectively) (Fig. 4). Interestingly, increasing egg and adult numbers were recorded after completely ceasing copulation induction methods from the $F_{11}$ generation (free-mating).

Based on observations made across 19 generations, $A n$. darlingi egg to adult development under laboratory conditions was on average $13.7 \pm 2.3$ days and ranged from $10\left(\mathrm{~F}_{10}\right)$ to $20\left(\mathrm{~F}_{5}\right)$ days. A longer egg to adult development ranging from 12 to 29 days was recorded for An. darlingi $\mathrm{F}_{1}$ reared under laboratory conditions [14], yet the average recorded for the colony lies within this range. Egg to larval development required two days. First instar larva to pupal development ranged from 6 to 16 days and pupal to adult development required two days. An additional file shows photos of the different stages of An. darlingi [see Additional file 3]. 


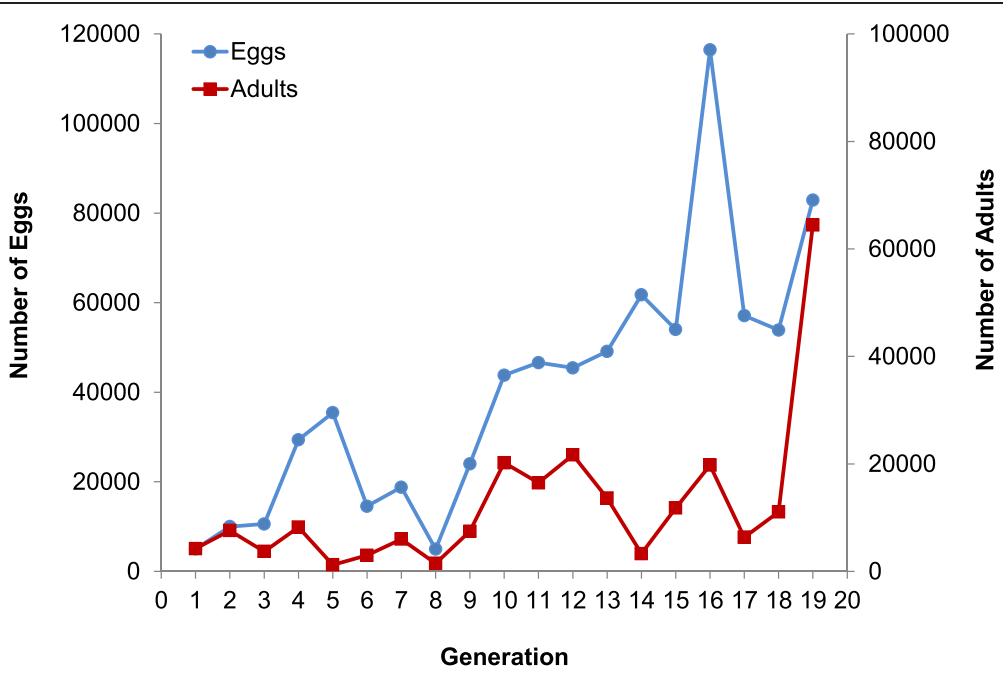

Fig. 4 Production of An. darlingi eggs and adults across 19 generations $\left(F_{1}-F_{12}\right)$ reared in the NAMRU-6 insectary in lquitos, Peru

\section{Discussion}

Herein it is reported the successful establishment of the first free-mating, highly productive, and long-lasting $A n$. darlingi colony by: a) inducing natural copulation through a combination of optimal thermo-period and light stimulation; and b) optimizing egg oviposition and larval rearing parameters. Despite the relevance of An. darlingi for malaria transmission in the Amazonian region [10, 11], many aspects of its physiology, behavior, ecology, genetics, and interaction with Plasmodium spp. are poorly understood due to the lack of a laboratory colony that can serve as a source of large numbers of individuals [35, 36]. This large and highly productive colony is a unique, valuable resource for developing, and evaluating effective vector- based strategies against malaria transmission in the Amazon. Extensive methodological details are provided to the scientific community to encourage the reproduction of the results, establishment of additional colonies, and extrapolation of the described methods to critical Anopheles vectors in the Amazon and in other regions of the world.

Historically the establishment of a laboratory colony of An. darlingi proved difficult, and this is the first report of a free-mating, autonomous colony of this important vector species. A recent study showed successful colonization of An. darlingi and P. vivax infection of colonized mosquitoes from $F_{3}$ to $F_{6}$ generations [15], yet no evidence of free-mating or a description of sexual behaviour was reported. Low sexual activity observed in adults confined to

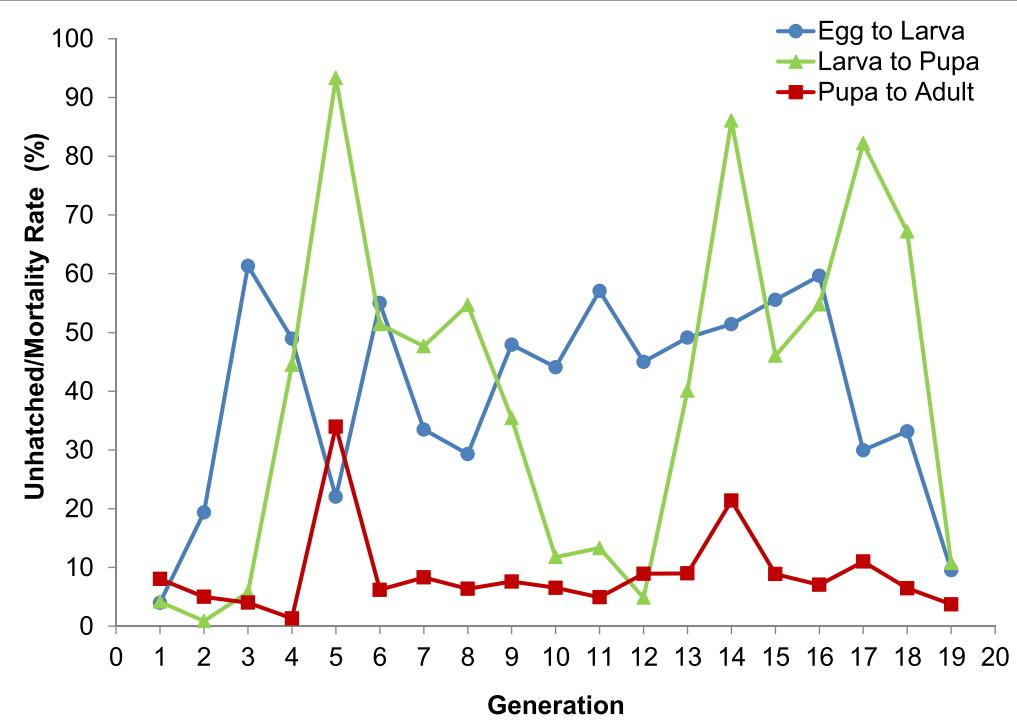

Fig. 5 Anopheles darlingi unhatched egg rates (\%), and larval, and pupal mortality (\%) across 19 generations $\left(F_{1}-F_{12}\right)$ reared in the NAMRU-6 insectary in lquitos, Peru. Free-mating was recorded from generation $F_{9}$ 
screened cages under insectary conditions has been the main cited obstacle in establishing an An. darlingi colony [23]. In this study it is shown that natural copulation occurs consistently and effectively in the laboratory with four-fold higher copulation rates in larger cages $(61 \times 61 \times 61 \mathrm{~cm})$. Additionally, external diurnal stimuli, was initially required to trigger sexual behaviour. This involved increased temperatures during the day and lower temperatures during twilight, in addition to exposure to a dim light beam at dusk, as to resemble field conditions. Light beams at dusk may resemble natural sunbeams decreasing at this time of the day, thus cueing mosquitoes that the sun is setting and potentially triggering their mating behaviour. These conditions sparked sexual activity in cages immediately after turning the lights off. In previous studies of $A n$. darlingi reproductive behaviour in the NAMRU-6 Insectary, a very small percentage of caged adult females were inseminated in the absence of any type of stimulus or when applying a light beam for 30-min during twilight [14]. In this study, a specific thermoperiod regime coupled to exposure to a LED beam resulted in a considerably higher mean insemination rate $(55 \%)$. This is in line with field studies where virgin females were released in the field at sunset, and when recaptured two hours later, $60 \%$ had been inseminated [20]. Sexual activity increased across generations from $2.6\left(\mathrm{~F}_{1}\right)$ to 40 $\left(\mathrm{F}_{14}\right)$ matings per minute. Light stimulation was not needed after the $F_{10}$ generation indicating the development and selection of a stenogamic colony.

It was also found that An. darlingi copulation rates were higher in 2 to 8 day-old mosquitoes, suggesting that the sexual stimulation method should be applied to that specific age range. Older females may not respond as effectively. Observations in colonized An. darlingi agree with those recorded in An. gambiae, for which the optimal age for male swarming in the field and female copulation success in the laboratory was found to be 4-8 days [37]. In the field, An. darlingi males probably fly at low altitudes, close to the vegetation, and remaining near breeding sites waiting to copulate, and even some may travel to food sources following females [20]. In this study, and in line with the hypothesized low flying altitude in the field, males were observed flying 5-40 $\mathrm{cm}$ above the cage floor, and forming a pseudoswarm, and apparently attracting females. Adults copulated while flying and fell to the cage floor with most mating pairs separating in less than second. All of these behaviors are similar to that of Neotropical Anopheles reared under laboratory conditions [22, 38]. Also, some apparent male competition for females was observed in this study, with two males attempting copulation simultaneously with the same female and all three falling together on the cage floor, which could have interfere with copulation. Further examination is needed to assess if this behaviour had an effect on copulation success.

Previously, an An. darlingi population from Lacandon Forest in Chiapas, Mexico was colonized by natural copulation induction and reared for nine generations. However, the colony collapsed after females stopped ovipositing in white containers placed inside cages [14]. In this study, this problem was prevented by using highlypreferred dark oviposition trays that perhaps better resemble natural oviposition sites. Native aquatic plants in oviposition trays served as resting places for ovipositing adults and larvae, but were removed from the rearing protocol as they appeared to be a water contamination source.

In addition to optimizing oviposition, an optimal larval feeding regime was established using commercially available rodent food. This food appears to meet the nutritional needs of the immature An. darlingi larvae in the laboratory, and has been tested in other Anopheles spp. effectively [22]. Other commercial animal foods (fish, dog, or monkey) appear to be not as suitable to rear $A n$. darlingi larvae possibly because their high fat content could lead to the formation of an oily/greasy layer on top of the water, which seems to kill larvae and promote the development of pathogenic microorganisms.

The establishment of autonomous neotropical anopheline colonies under insectary conditions has had variable success. Some anopheline species are considered relatively easy to colonize, such as Anopheles albimanus that does not require copulation induction [38]. In contrast, An. pseudopunctipennis requires artificial induction of mating [22]. In addition, the number of generations needed to develop an autonomous colony also varies by species and population origin. For example, the Tapachula, and Abasolo Mexican laboratory strains of An. pseudopunctipennis required 5 and 12 generations to select a free-mating population, respectively [22]. A stenogamous colony of Anopheles albitarsis from Brazil was obtained in six generations [39] while free-mating in Anopheles aquasalis was recorded in the $\mathrm{F}_{2}$ generation [40]. Finally, the previously attempted Mexican An. darlingi colony collapsed at the $\mathrm{F}_{9}$ generation before becoming autonomous [14], yet the Peruvian An. darlingi colony became autonomous after nine generations. Therefore, it is suggested that free-mating in colonized An. darlingi can only be demonstrated after rearing at least nine generations.

The fact that this study reports the first establishment of a stenogamic laboratory colony of An. darlingi despite many previous attempts $[14,16,19]$ proves that colonizing and rearing this species is very challenging. The An. darlingi colonization approach used involved three critical steps: 1) employment of a very rigorous yet replicable procedure to trigger sexual behavior leading to successful insemination, 2) optimization of proper conditions for 
consistent oviposition, and 3) standardization of an adequate immature feeding and rearing regime. This approach produced a practical method to establish $A n$. darlingi colonies and represents an alternative to the time-consuming and unpractical forced-mating method. The overall approach can be easily adapted and replicated for the establishment of other An. darlingi colonies, and could be useful to establish colonies of other critical malaria vectors worldwide.

Unlimited access to An. darlingi colony material will allow us to examine and understand unknown biological aspects of this important malaria vector including longevity, gonotrophic cycle length, oviposition behaviour, fecundity and so forth, as well as behavioural, and genetic traits that can be exploited for the development of novel and effective vector control approaches. It is also important to better understand male traits that determine copulation success, such as body size, nutritional status, and any associated genetic components, to facilitate future release of genetically altered males refractory to Plasmodium spp. Additionally, the interaction of $A n$. darlingi with Plasmodium spp. parasites under controlled laboratory conditions and the performance of novel drug therapies targeting parasite development in the mosquito can now be better examined. Preliminary $P$. vivax infection studies via membrane feeding assays (4 successful experimental infections) conducted in colonized $A n$. darlingi adult females from the $\mathrm{F}_{19}-\mathrm{F}_{21}$ generations have shown that these colonized mosquitoes are susceptible to $P$. vivax infection, with number of oocysts per mosquito ranging from 2 to 197 (mean $\pm \mathrm{SD}=38.6 \pm$ 41.5 ), and number of sporozoites per mosquito ranging from 152 to 61,867 (mean $\pm S D=8,141.4 \pm 9,489.7$ ). These values are comparable with those reported for colonized An. darlingi $\left(\mathrm{F}_{1}-\mathrm{F}_{6}\right)$ experimentally infected with $P$. vivax, with oocyst number per mosquito ranging from 1 to 57 and sporozoite number per mosquito ranging from 150 to 7,380 [15]. These preliminary results will be confirmed by additional $P$. vivax experimental infections of colonized An. darlingi from the $\mathrm{F}_{22}-\mathrm{F}_{26}$ generations. Overall, information gathered from physiological, behavioural, and experimental infection studies conducted in colonized $A n$. darlingi will be useful in developing strategies for comprehensive, integrated malaria vector control.

\section{Conclusions}

Herein it is provided the first report of a well-established, highly productive, stenogamous colony of An. darlingi, having produced a total of 231,591 adults in 19 generations. Mating behavior of An. darlingi under laboratory conditions is described for the first time. Methods developed here may well prove useful to start additional $A n$. darlingi colonies and in the establishment of other difficult malaria vector species. Having an established $A n$. darlingi laboratory colony represents an invaluable resource for critical malaria research in the Amazon region.

\section{Additional files}

\section{Additional file 1: Video of natural copulation under laboratory} conditions in An. darlingi $\mathrm{F}_{1}$ adults (A) and $\mathrm{F}_{14}$ adults (B).

Additional file 2: Anopheles darlingi rearing rooms in the NAMRU-6 insectary in lquitos.

Additional file 3: Developmental stages of An. darlingi.

\section{Competing interests}

The authors declare that they have no competing interests.

\section{Authors' contributions}

CVT designed, supervised, and performed the experiments, interpreted data, and drafted the manuscript. GMV designed and supervised the experiments, analyzed and interpreted data, and drafted the manuscript. VLS organized field operations to collect wild mosquito populations, identified anophelines collected during the study, supervised colony rearing, and participated in manuscript revision. KEV and AHR conducted mosquito rearing, performed natural copulation induction experiments, entered data, and participated in manuscript revision. YML confirmed the molecular identification of the An. darlingi colony and participated in manuscript revision. CFM participated in study design, data interpretation, and manuscript revision. AGL participated in data analysis, data interpretation, and manuscript revision. FMS participated in study design and manuscript revision. All authors read and approved the final manuscript.

\section{Acknowledgements}

We would like to thank the Ministerio de Agricultura y Riego de Perú, Dirección General Forestal y de Fauna Silvestre, and the Dirección de Salud, Gobierno Regional de Loreto for permission to conduct these studies: Anopheles darlingi collections in Loreto were conducted under the auspices of Resolución Directoral No. 0406-2013-MINAGRI-DGFFS/DGEFFS; An. darlingi molecular identification was conducted under the auspices of Contrato de Acceso Marco a Recursos Geneticos No. 0017-2014-MINAGRI-DGFFS/DGEFFS. We would like to thank Fanny Castro, Geidin Chavez, Hugo Jaba, Luz Romero, Miguel Vásquez, and Alex Vásquez for technical support. Additionally, we would like to thank Dr. Craig Stoops for providing helpful comments on the manuscript. Financial support was provided by the Global Emerging Infections Surveillance and Response System (AFHSC/GEIS) of the U.S. Department of Defense sustainment funding award (FMS, GMV), the Consejo Nacional de Ciencia y Tecnología (CONACYT) México, grant CB2008-105806-M (CVT), and by the training grant 2D43 TW007393-06 awarded to NAMRU-6 by the Fogarty International Center of the U.S. National Institutes of Health. YML was supported by the National Research Council (NRC) Research Associate Program.

\section{Disclaimer}

The views expressed in this article are those of the authors and do not necessarily reflect the official policy or position of the Department of the Navy, Department of Defense, U.S. Army, or the U.S. Government.

\section{Copyright statement}

Several authors of this manuscript are military service members or employees of the U.S. Government. This work was prepared as part of their duties. Title 17 U.S.C. $\S 105$ provides that 'Copyright protection under this title is not available for any work of the United States Government.' Title 17 U.S.C. § 101 defines a U.S. Government work as a work prepared by a military service member or employee of the U.S. Government as part of that person's official duties.

\section{Author details}

${ }^{1}$ Centro Regional de Investigación en Salud Pública/Instituto Nacional de Salud Pública (CRISP/INSP), Tapachula, Chiapas, Mexico. ${ }^{2}$ Department of Entomology, U.S. Naval Medical Research Unit No. 6 (NAMRU-6), Bellavista, Callao, Peru. ${ }^{3}$ Walter Reed Biosystematics Unit, Museum Support Center, Smithsonian Institution, Suitland, MD, USA. "Walter Reed Army Institute of Research, Silver Spring, MD, USA. ${ }^{5}$ Department of Entomology, Smithsonian 
Institution, Washington, DC, USA. ${ }^{6}$ Department of Parasitology, U.S. Naval Medical Research Unit No. 6 (NAMRU-6), Bellavista, Callao, Peru.

Received: 24 December 2014 Accepted: 19 May 2015 Published online: 30 May 2015

\section{References}

1. Hiwat H, Bretas G. Ecology of Anopheles darlingi Root with respect to vector importance: a review. Parasit Vectors. 2011:4:177.

2. Sinka ME, Rubio-Palis Y, Manguin S, Patil AP, Temperley WH, Gething PW et al. The dominant Anopheles vectors of human malaria in the Americas: occurrence data, distribution maps and bionomic précis. Parasit Vectors. 2010;3:72.

3. Vittor AY, Gilman RH, Tielsch J, Glass G, Shields T, Lozano WS, et al. The effect of deforestation on the human-biting rate of Anopheles darlingi, the primary vector of falciparum malaria in the Peruvian Amazon. Am J Trop Med Hyg. 2006;74:3-11.

4. Vittor AY, Pan W, Gilman RH, Tielsch J, Glass G, Shields T, et al. Linking deforestation to malaria in the Amazon: characterization of the breeding habitat of the principal malaria vector, Anopheles darlingi. Am J Trop Med Hyg. 2009;81:5-12

5. Lounibos LP, Conn JE. Malaria vector heterogeneity in South America. Am Entomol. 2000;46:238-49.

6. Moutinho PR, Gil LH, Cruz RB, Ribolla PE. Population dynamics, structure and behavior of Anopheles darlingi in a rural settlement in the Amazon rainforest of Acre, Brazil. Malar J. 2011;10:174.

7. Zimmerman RH, Lounibos LP, Nishimura N, Galardo AK, Galardo CD, Arruda ME. Nightly biting cycles of malaria vectors in a heterogeneous transmission area of eastern Amazonian Brazil. Malar J. 2013;12:262.

8. Naranjo-Diaz N, Rosero DA, Rua-Uribe G, Luckhart S, Correa MM. Abundance, behavior and entomological inoculation rates of anthropophilic anophelines from a primary Colombian malaria endemic area. Parasit Vectors. 2013;6:61.

9. Flores-Mendoza C, Fernandez R, Escobedo-Vargas KS, Vela-Perez Q, Schoeler GB. Natural Plasmodium infections in Anopheles darlingi and Anopheles benarrochi (Diptera: Culicidae) from eastern Peru. J Med Entomol. 2004:41:489-94.

10. Aramburu GJ, Ramal AC, Witzig R. Malaria reemergence in the Peruvian Amazon region. Emerg Infect Dis. 1999;5:209-15.

11. Roper MH, Torres RS, Goicochea CG, Andersen EM, Guarda JS, Calampa C, et al. The epidemiology of malaria in an epidemic area of the Peruvian Amazon. Am J Trop Med Hyg. 2000;62:247-56.

12. Reinbold-Wasson DD, Sardelis MR, Jones JW, Watts DM, Fernandez R, Carbajal F, et al. Determinants of Anopheles seasonal distribution patterns across a forest to periurban gradient near lquitos, Peru. Am J Trop Med Hyg. 2012;86:459-63.

13. Parker BS, Paredes Olortegui M, Peñataro Yori P, Escobedo K, Florin D, Rengifo Pinedo $\mathrm{S}$, et al. Hyperendemic malaria transmission in areas of occupation-related travel in the Peruvian Amazon. Malar J. 2013;12:178.

14. Villarreal-Treviño C, Arredondo-Jimenez Jl, Malo IR, Rodriguez MH. Colonization of Anopheles darlingi from the Lacandon Forest, Chiapas, Mexico [Abstract]. J Am Mosq Control Assoc. 2001;12:LB0220-1.

15. Moreno M, Tong C, Guzmán M, Chuquiyauri R, Llanos-Cuentas A, Rodriguez $\mathrm{H}$, et al. Infection of laboratory-colonized Anopheles darlingi mosquitoes by Plasmodium vivax. Am J Trop Med Hyg. 2014;90:612-6.

16. Giglioli G. Laboratory colony of Anopheles darlingi. J Natl Malar Soc. 1947:6:159-64.

17. Bates M. The laboratory colonization of Anopheles darlingi. J Natl Malar Soc 1947;6:155-8.

18. Freire SA, Farías GS. Criacão e alguns dados sôbre a biología do Anopheles (N.) darlingi. Rev Bras Biol. 1947;7:57-66.

19. Buralli GM, Bergo ES. Manutenção de colônia de Anopheles darlingi Root, 1926, em laboratório. Rev Inst Med Trop Sao Paulo. 1988:30:157-64.

20. Lounibos LP, Lima DC, Lourenço-de-Oliveira R. Prompt mating of released Anopheles darlingi in western Amazonian Brazil. J Am Mosq Control Assoc. 1998;14:210-3

21. López-Sifuentes VM. Bioecología Reproductiva de Anopheles (Nyssorhynchus) darlingi Root 1926, Vector Principal de Malaria en la Cuenca Amazónica Peruana, Tesis de Maestría para la obtención del grado de Magister en Ciencias con mención en Ecología y Desarrollo Sostenible. Iquitos, Perú: Facultad de Ciencias Biológicas, Universidad Nacional de la Amazonia Peruana; 2013.
22. Villarreal-Treviño C, Arredondo-Jimenez Jl, Rodriguez MH, Ulloa A. Colonization of Anopheles pseudopunctipennis from Mexico. J Am Mosq Control Assoc. 1998;14:369-72.

23. Lima JB, Valle D, Peixoto AA. Adaptation of a South American malaria vector to laboratory colonization suggests faster-male evolution for mating ability. BMC Evol Biol. 2004;4:12.

24. Baker RH, French WL, Kitzmiller JB. Induced copulation in Anopheles mosquitoes. Mosq News. 1962;22:16-7.

25. WHO. Manual on practical entomology in malaria. Part II: Methods and Techniques. WHO Division of Malaria and Other Parasitic diseases. Geneva: World Health Organization; 1975

26. Branch O, Casapia WM, Gamboa DV, Hernandez JN, Alava FF, Roncal N, et al Clustered local transmission and asymptomatic Plasmodium falciparum and Plasmodium vivax malaria infections in a recently emerged, hypoendemic Peruvian Amazon community. Malar J. 2005:4:27.

27. Bharti AR, Chuquiyauri R, Brouwer KC, Stancil J, Lin J, Llanos-Cuentas A, et al. Experimental infection of the neotropical malaria vector Anopheles darlingi by human patient-derived Plasmodium vivax in the Peruvian Amazon. Am J Trop Med Hyg. 2006;75:610-6.

28. Faran ME, Linthicum KJ. A handbook of the Amazonian species of Anopheles (Nyssorhynchus) (Diptera: Culicidae). Mosq Syst. 1981;13:1-81.

29. Consoli Rotraut AGB, Lourenço-de-Oliveira R. Principais mosquitos de importância sanitaria no Brasil. Brasil: Editora FIOCRUZ; 1994.

30. Linton YM, Pecor JE, Porter CH, Mitchell LB, Garzón-Moreno A, Foley DH et al. Mosquitoes of eastern Amazonian Ecuador: biodiversity, bionomics and barcodes. Mem Inst Oswaldo Cruz. 2013;108 Suppl 1:100-9.

31. Briegel H. Fecundity, metabolism, and body size in Anopheles (Diptera: Culicidae) vectors of malaria. J Med Entomol. 1990;27:839-50.

32. Lyimo EO, Takken W. Effects of adult body size on fecundity and the pre-gravid rate of Anopheles gambiae females in Tanzania. Med Vet Entomol. 1993;7:328-32.

33. Ratnasingham S, Hebert PDN. BOLD: The Barcode of Life Data System (www.barcodinglife.org). Mol Ecol Notes. 2007;7:355-64.

34. Moreno M, Marinotti O, Krywinski J, Tadei WP, James AA, Achee NL. Research complete mtDNA genomes of Anopheles darlingi and an approach to anopheline divergence time. DNA. 2010;8:14.

35. Marinotti O, Cerqueira GC, de Almeida LG, Ferro MI, Loreto EL, Zaha A, et al. The genome of Anopheles darlingi, the main neotropical malaria vector. Nucleic Acids Res. 2013;41:7387-400.

36. Molina-Cruz A, Barillas-Mury C. The remarkable journey of adaptation of the Plasmodium falciparum malaria parasite to New World anopheline mosquito. Mem Inst Oswaldo Cruz. 2014:5:662-7.

37. Sawadogo SP, Diabaté A, Toé HK, Sanon A, Lefevre T, Baldet T, et al. Effects of age and size on Anopheles gambiae s.s. male mosquito mating success. J Med Entomol. 2013;50:285-93.

38. Ramsey JM, Salinas E, Lopez JR, Del Angel-Cabañas G, Martinez L, Bown DN. Laboratory oviposition, fecundity and egg hatching ability of colonized Anopheles albimanus from southwestern Mexico. J Am Mosq Control Assoc. 1988:4:509-15.

39. Horosko III S, Lima JBP, Brandolini MB. Establishment of a free-mating colony of Anopheles albitarsis from Brazil. J Am Mosq Control Assoc. 1997;13:95-6.

40. Da Silva ANM, Dos Santos CCB, Lacerda RNL, Santa Rosa EP, De Souza RTL, Galiza D, et al. Laboratory colonization of Anopheles aquasalis (Diptera: Culicidae) in Belém, Pará, Brazil. J Med Entomol. 2006:43:107-9. 